\title{
The effect of probiotic supplementation on the gut microbiota of preterm infants
}

\author{
Erica L. Plummer ${ }^{1,2,3, *}$, Jennifer A. Danielewski ${ }^{1,2}$, Suzanne M. Garland ${ }^{1,2,4}$, Jenny Su ${ }^{1,2}$, Susan E. Jacobs ${ }^{4,5,6}$ and Gerald \\ L. Murray ${ }^{1,2,4}$, on behalf of The ProPrems Study Group
}

\begin{abstract}
Introduction. Probiotic supplementation of preterm infants may prevent serious morbidities associated with prematurity.

Aim. To investigate the impact of probiotic supplementation on the gut microbiota and determine factors associated with detection of probiotic species in the infant gut.

Hypothesis/Gap Statement. Probiotic supplementation increases the long-term colonization of probiotic species in the gut of preterm infants.

Methodology. Longitudinal stool samples were collected from a cohort of very preterm infants participating in a blinded randomized controlled trial investigating the impact of probiotic supplementation (containing Bifidobacterium longum subsp. infantis BB-02, Bifidobacterium animalis subsp. lactis BB-12 and Streptococcus thermophilus TH-4) for prevention of late-onset sepsis. The presence of $B$. longum subsp. infantis, B. animalis subsp. lactis and $S$. thermophilus was determined for up to 23 months after supplementation ended using real-time PCR. Logistic regression was used to investigate the impact of probiotic supplementation on the presence of each species.
\end{abstract}

Results. Detection of B. longum subsp. infantis [odds ratio (OR): $53.1 ; 95 \%$ confidence interval (CI): $35.6-79.1 ; P<0.001$ ], B. animalis subsp. lactis (OR: 89.1; $95 \% \mathrm{Cl}: 59.0-134.5 ; P<0.001$ ) and S. thermophilus (OR: $5.66 ; 95 \% \mathrm{Cl}: 4.35-7.37 ; P<0.001$ ) was increased during the supplementation period in infants receiving probiotic supplementation. Post-supplementation, probiotic-supplemented infants had increased detection of $B$. longum subsp. infantis (OR: 2.53; 95\% Cl: 1.64-3.90; $P<0.001$ ) and B. animalis subsp. lactis (OR: 1.59; $95 \% \mathrm{Cl}: 1.05-2.41 ; P=0.030$ ). Commencing probiotic supplementation before 5 days from birth was associated with increased detection of the probiotic species over the study period (B. longum subsp. infantis, OR: 1.20; B. animalis subsp. lactis, OR: 1.28; S. thermophilus, OR: 1.45).

Conclusion. Probiotic supplementation with B. longum subsp. infantis BB-02, B. animalis subsp. lactis BB-12 and S. thermophilus $\mathrm{TH}-4$ enhances the presence of probiotic species in the gut microbiota of very preterm infants during and after supplementation. Commencing probiotic supplementation shortly after birth may be important for improving the long-term colonization of probiotic species.

Received 26 February 2021; Accepted 10 June 2021; Published 25 August 2021

Author affiliations: ${ }^{1}$ Centre for Women's Infectious Diseases Research, The Royal Women's Hospital, Parkville, Victoria, Australia; ${ }^{2}$ nfection \& Immunity Theme, Murdoch Children's Research Institute, Melbourne, Victoria, Australia; ${ }^{3}$ Central Clinical School, Monash University, Melbourne, VIC, Australia; ${ }^{4}$ Department of Obstetrics and Gynaecology, University of Melbourne, Parkville, Victoria, Australia; ${ }^{5}$ Neonatal Services, The Royal Women's Hospital, Parkville, Victoria, Australia; ${ }^{\circ}$ Clinical Sciences, Murdoch Children's Research Institute, Melbourne, Victoria, Australia.

*Correspondence: Erica L. Plummer, erica.plummer@mcri.edu.au

Keywords: Bifidobacterium longum subsp. infantis; Bifidobacterium animalis subsp. lactis; gut microbiota; preterm infants; probiotics; Streptococcus thermophilus.

Abbreviations: $\mathrm{Cl}$, confidence interval; GEE, generalized estimating equation; IQR, interquartile range; LOS, late-onset sepsis; NEC, necrotizing enterocolitis; OR, odds ratio; PCR, polymerase chain reaction; RCT, randomized control trial.

Raw sequencing data used in this study have been previously deposited in NCBI's Short Read Archive (SRA) under BioProject PRJNA353658, https:// www.ncbi.nlm.nih.gov/bioproject/PRJNA353658/.

One supplementary figure and three supplementary tables are available with the online version of this article. $001403 \odot 2021$ The Authors 
Table 1. Primers and probes used in this study

\begin{tabular}{|c|c|c|}
\hline Target & Primer sequence $\left(5^{\prime}-3^{\prime}\right)^{*}$ & Reference \\
\hline \multirow{5}{*}{$\begin{array}{l}\text { B. longum } \\
\text { subsp. infantis }\end{array}$} & F: TTCCAGTTGATCGCATGGTC & {$[31]$} \\
\hline & R: GGAAACCCCATCTCTGGGAT & \\
\hline & P: CY5- & [13] \\
\hline & TCAAgCCCAggTAAggTTCTTCgC & \\
\hline & BHQ3 & \\
\hline \multirow{5}{*}{$\begin{array}{l}\text { B. animalis } \\
\text { subsp. lactis }\end{array}$} & F: GTGGAGACACGGTTTCCC & [32] \\
\hline & $\mathrm{R} \dagger:$ CACACCACACAATCCAATAC & \\
\hline & P: FAM- & [13] \\
\hline & TTCACAGGTGGTGCATGGTCGT & \\
\hline & BHQ1 & \\
\hline \multirow[t]{4}{*}{ S. thermophilus } & F: TTATTTGAaAGgGGCaATTGCT & {$[13,27]$} \\
\hline & R: GTGAACttTCCACTCtCACAC & Modified \\
\hline & P: CY5-ACTACAAGATGGACCTGCGT & \\
\hline & BHQ3 & \\
\hline \multirow{3}{*}{$\begin{array}{l}\text { All } \\
\text { Bifidobacterium }\end{array}$} & F†: GGGATGCTGGTGTGGAAGAGA & {$[14]$} \\
\hline & R†: TGCTCGCGTCCACTATCCAGT & \\
\hline & $\begin{array}{c}\mathrm{P} \uparrow: \text { FAM-TCAAACCACCACGCGCCA } \\
\text { BHQ1 }\end{array}$ & \\
\hline
\end{tabular}

*Lower case letters indicate locked nucleic acid (LNA) bases.

†Primers/probe is located within the 16S-23S rRNA intergenic spacer region; all other primers and probes are located in the $16 \mathrm{~S}$ rRNA gene region, which is more highly conserved.

$F$, forward primer; $R$, reverse primer; $P$, probe; FAM,

6-carboxyfluorescein; Cy5, cyanine 5; BHQ, Black Hole Quencher.

\section{INTRODUCTION}

Preterm infants have an altered gastrointestinal microbiota compared with term infants, including reduced species diversity, reduced numbers of Bifidobacterium species and Lactobacillus species, and higher numbers of potentially pathogenic Enterobacteriaceae and Clostridium difficile $[1,2]$. These differences probably result from reduced exposure to maternal microbiota, from immaturity of the intestinal immune response, and from the various medical interventions necessary in management within neonatal intensive care units [3].

Probiotics are defined by the World Health Organisation as 'live microorganisms which when administered in adequate amounts confer a health benefit on the host' [4]. Recent systematic reviews and meta-analyses have reported that probiotic supplementation is effective for the prevention of serious morbidities associated with prematurity, including mortality, late-onset sepsis (LOS) and necrotizing enterocolitis (NEC) [5-8]. The ProPrems randomized controlled trial (RCT) was a large multi-centre double blind, placebocontrolled randomized trial investigating the effect of supplementation with a probiotic combination (Bifidobacterium longum subsp. infantis strain BB-02, Streptococcus thermophilus strain TH-4 and Bifidobacterium animalis subsp. lactis strain BB-12, containing a total of $1 \times 10^{9}$ organisms) on LOS in very preterm infants $[9,10]$. A secondary objective of the ProPrems RCT was to investigate the impact of the probiotic combination on the incidence of NEC. The ProPrems RCT found that while administration of the probiotic was not associated with reduction in LOS or mortality, it resulted in a $54 \%$ reduced risk of NEC of Bell stage 2 or more [10].

The exact mechanism(s) by which probiotics exert benefits in preterm infants is not known and may be strain-specific. One likely mechanism is modulation of gut microbiota composition [11]. We previously reported findings from a 16S rRNA gene pyrosequencing study of 66 infants who participated in the ProPrems RCT [12]. We found that infants who received probiotic were more likely to have increased abundance of Bifidobacterium in their gut microbiota during the supplementation period compared to infants randomized to placebo; however, no difference in the abundance of Streptococcus was observed [12]. This study was limited in that it only provided genus-level resolution and thus could not identify if the elevated bifidiobacteria were subspecies found in the probiotic combination. In the present study, we investigated the impact of probiotic supplementation on detection of B. longum subsp. infantis, B. animalis subsp. lactis and S. thermophilus in the gut microbiota of a subset of ProPrems infants using real-time PCR (qPCR). We also determined concordance of the 16S rRNA gene sequencing data [12] and qPCR for the detection of Bifidobacterium.

\section{METHODS}

\section{Specimen collection and laboratory methods}

The ProPrems RCT protocol and details on specimen collection have been reported elsewhere $[9,10,12]$. Briefly, preterm infants born weighing less than $1500 \mathrm{~g}$ and less than 32 weeks of gestation were randomized to receive either a probiotic combination at $1.5 \mathrm{~g}$ per day $\left[1 \times 10^{9}\right.$ total organisms of B. longum subsp. infantis BB-02 $\left(300 \times 10^{6}\right)$, S. thermophilus TH-4 $\left(350 \times 10^{6}\right)$ and B. animalis subsp. lactis BB-12 $\left(350 \times 10^{6}\right.$; ABC Dophilus Probiotic Powder for Infants; Solgar), in a maltodextrin base powder] or placebo (maltodextrin powder). Infants commenced the assigned intervention when they were tolerating at least $1 \mathrm{ml}$ of milk feeds every $4 \mathrm{~h}$. Infants continued to receive the intervention until discharge home or term-corrected age, whichever came first. Stool swabs were collected from Victorian infants as close to the following time points as possible: prior to commencement of the study powder, after 1, 4 and 8 weeks of study powder, and at 6 and 12 months of age corrected for prematurity. An anal swab was collected if the infant had not yet passed a stool, and a swab of the first meconium was collected where available.

Swabs were rotated in $400 \mu \mathrm{l}$ of PBS and extracted using the MagNA Pure 96 nucleic acid extraction system (Roche Diagnostics) and the MagNA Pure 96 DNA and viral small volume Isolation Kit, as previously described [13]. Extracted DNA was tested using qPCR assays targeting the $16 \mathrm{~S}$ rRNA gene region for the detection of $B$. longum subsp. infantis, $B$. animalis subsp. lactis, S. thermophilus $[9,13]$, and all Bifidobacterium targeting the 16S-23S rRNA intergenic spacer region [14] (Table 1). PCR 
and cycling conditions were as previously outlined $\left(95^{\circ} \mathrm{C}\right.$ for 10 min followed by 50 cycles of $95^{\circ} \mathrm{C}$ for $10 \mathrm{~s}$ and $60^{\circ} \mathrm{C}$ for $55 \mathrm{~s}$ ) [13]. PCR cycling conditions for all four qPCR assays were the same, allowing 22 samples (plus one negative and one positive control per assay) to be amplified for all four targets in one run on the LightCycler 480 real-time instrument (Roche Diagnostics). Samples were analysed in four subsets of 24 (a subset for each assay) using the second derivative maximum method to obtain the quantification cycle (Cq) for each target. As a positive control, $1.55 \mathrm{~g}$ of ABC Dophilus Probiotic Powder for Infants was resuspended in $3.62 \mathrm{ml}$ PBS, from which $200 \mu \mathrm{l}$ was extracted as described [13]. A serial dilution of the calculated copies per organism was performed, and the dilution estimated at 1000 copies per $5 \mu \mathrm{l}$ was used as a positive control. The methodology for sample preparation for 454 pyrosequencing (using a Roche 454 Genome Sequencer instrument; GS FLX Titanium Chemistry), sequence processing and taxonomic assignment has been previously reported [12]. Briefly, gut microbiota composition was determined for 215 specimens using bifidobacteria-optimized PCR primers [15] that amplify the V3-V5 hypervariable regions of the 16S rRNA gene. Sequencing data were processed with QIIME (Version 1.8.0) [16], using UCLUST (Version 1.2.22q) [17] to cluster sequences into operational taxonomic units using a similarity threshold of $97 \%$. The UCLUST consensus taxonomy assigner (Version 1.2.22q) [17] was used to assign taxonomy using default parameters and the sILVA reference database (Version 111) [18].

\section{Statistical analysis}

The qPCR data were initially expressed as a binary variable, i.e. detected $(\mathrm{Cq} \leq 45)$ or not detected $(\mathrm{Cq}>45)$. Logistic regression models that accounted for repeated measures within infants were fitted using generalized estimating equations (GEEs) to investigate the association between probiotic supplementation and detection of B. longum subsp. infantis, B. animalis subsp. lactis and $S$. thermophilus in the gut microbiota. We grouped specimens into three categories based on timing of collection and investigated the association between probiotic supplementation and detection of $B$. longum subsp. infantis, B. animalis subsp. lactis and $S$. thermophilus in the gut microbiota before, during and after supplementation. Logistic regression was also used to determine what factors were associated with detection of probiotic species in infants randomized to the probiotic group.

Bacterial abundance of $B$. longum subsp. infantis, B. animalis subsp. lactis and $S$. thermophilus was approximated using $\mathrm{Cq}$ values and summarized using box plots. A low Cq value indicates a higher copy number of the target organism than high Cq values. Specimens with no detectable target were assigned a Cq value of 45. Differences in Cq values between probiotic and placebo infants at the three time points (i.e. before, during and after supplementation) were assessed using linear regression models fitted using GEEs.

Sequencing data for Bifidobacterium were expressed as a binary variable. Specimens were classified as detected if they had any sequence reads assigned to Bifidobacterium and not detected if there were no sequence reads assigned. Concordance of Bifidobacterium detection using 16S rRNA gene sequencing and Bifidobacterium detected using the genus-specific qPCR assay was determined using Cohen's kappa statistic.

All analyses were carried out using STATA (Version 14; StataCorp).

\section{RESULTS}

A total of 683 infants were recruited to the ProPrems trial at hospitals located in Victoria, Australia. Specimens were collected from 680 infants and qPCR data were available for 472 infants. Thirteen infants and their specimens were excluded because they did not receive the allocated intervention. As a result, 459 infants contributed 2335 specimens to this analysis. This included 1181 specimens from 229 infants randomized to the probiotic combination and 1154 specimens from 230 infants randomized to the placebo. A median of five specimens [interquartile range (IQR): 4-6] were collected from infants. Participant characteristics are summarized in Table 2. Two-thirds of infants were born by Caesarean section (68\%) and 28 infants developed NEC during the study. One infant developed NEC at 5 months of age after their participation in the study ended. Characteristics were similar between the allocation groups, except that more infants were born at $\leq 28$ weeks of gestation in the placebo group [ $47,95 \%$ confidence interval (CI): 40-53\%] compared to the probiotic group (35\%, 95\% CI: $39-42 \%)$.

Over the whole study period, infants randomized to probiotic were more likely to have $B$. animalis subsp. lactis [odds ratio (OR): 10.2; 95\% CI: 8.35-12.5; $P<0.001$, Table 3], B. longum subsp. infantis (OR: 7.16; 95\% CI: 5.89-8.69; $P<0.001$ ) and S. thermophilus (OR: 2.64; 95\% CI: $2.25-3.11 ; P<0.001$ ) detected than infants randomized to placebo.

A total of 679 specimens were collected prior to starting supplementation [median age of collection $=2$ days (range $=0$ 15); Table S1, available in the online version of this article]. There was no significant difference in the detection of B. longum subsp. infantis, B. animalis subsp. lactis and $S$. thermophilus before supplementation started between the probiotic and placebo groups (Table 3). A total of 1251 specimens were collected during the supplementation period (median age of collection $=30$ days, range $=0-96$ days; Table S1). Detection of B. longum subsp. infantis (OR: $53.1 ; 95 \%$ CI: 35.6-79.4; $P<0.001$ ), B. animalis subsp. lactis (OR: 89.1; 95\% CI: $59.0-134.5 ; P<0.001$ ) and S. thermophilus (OR: 5.66 ; 95\% CI: 4.35-7.37; $P<0.001)$ was increased during the supplementation period in infants receiving probiotic. A total of 401 specimens were collected post-supplementation [median age of collection $=275$ days (range $=13-731$ ); collected a range of 1 day to 23 months after ending supplementation], with the majority of post-supplementation specimens collected 6-12 months after the last dose of powder ( $n=203 / 401,51 \%$; Table S1). Post-supplementation, infants who received probiotic had increased detection of $B$. longum subsp. infantis (OR: 2.53; 
Table 2. Participant characteristics

\begin{tabular}{|c|c|c|c|}
\hline Characteristic & qPCR sub-study participants $(N=459)$ & Probiotic $(N=229)$ & Placebo $(N=230)$ \\
\hline \multicolumn{4}{|l|}{ < 28 weeks, $n(\%)$} \\
\hline No & $271(59)$ & $148(65)$ & $123(53)$ \\
\hline Yes & $188(41)$ & $81(35)$ & $107(47)$ \\
\hline \multicolumn{4}{|l|}{$<1000 \mathrm{~g}, n(\%)$} \\
\hline No & $258(56)$ & $131(57)$ & $127(55)$ \\
\hline Yes & $201(44)$ & $98(43)$ & $103(45)$ \\
\hline \multicolumn{4}{|l|}{ Male, $n(\%)$} \\
\hline \multicolumn{4}{|l|}{ Caesarean delivery, $n(\%)$} \\
\hline No & $145(32)$ & $70(31)$ & $75(33)$ \\
\hline Yes & $314(68)$ & $159(69)$ & $155(67)$ \\
\hline \multicolumn{4}{|l|}{ Any NEC, $n(\%)$} \\
\hline No & $430(94)$ & $218(95)$ & $212(92)$ \\
\hline Yes & $29(6)$ & $11(5)$ & $18(8)$ \\
\hline $\begin{array}{l}\text { Age commenced study powder, days, median } \\
\text { (IQR) }\end{array}$ & $5(4-7)$ & $5(4-7)$ & $5(4-7)$ \\
\hline Post-supplementation & 401,259 & 207,132 & 194,127 \\
\hline
\end{tabular}

$I Q R$, interquartile range; NEC, necrotizing enterocolitis.

$95 \%$ CI: $1.64-3.90 ; P=0.030)$ and $B$. animalis subsp. lactis (OR: 1.59; 95\% CI: 1.05-2.41; $P<0.001$ ), but no difference in detection of $S$. thermophilus (OR: 1.40; 95\% CI: 0.76-2.57; $P=0.283$ ).

We observed no difference in the Cq value of B. longum subsp. infantis, B. animalis subsp. lactis and $S$. thermophilus between randomization groups prior to supplementation (Fig. S1 and Table S2). During the supplementation period, the Cq value of the three probiotic species was lower (indicating higher abundance) in infants receiving probiotic compared to placebo infants [B. longum subsp. infantis median Cq value $=22$ (IQR: 19, 27) vs. 45 (IQR: 45, 45), coefficient $=-16.63$, 95\% CI: $-17.68,-15.58 ; P<0.001, B$. animalis subsp. lactis median Cq value $=32$ (IQR: 28, 36) vs. 45 (IQR: 45, 45), coefficient $=-11.70,95 \%$ CI: $-12.29,-11.09 ; P<0.001$ and $S$. thermophilus median Cq value=45 (IQR: 40, 45) vs. 45 (IQR: 45,
45), coefficient $=-1.41,95 \%$ CI: $-1.92,-0.89 ; P<0.001]$. Postsupplementation we observed lower Cq values of B. longum subsp. infantis in probiotic-supplemented infants [median Cq value $=31$ (IQR: 23,45$)$ vs. 45 (IQR: 28,45$)$, coefficient $=-4.69$, $95 \% \mathrm{CI}:-6.77,-2.61 ; P<0.001]$, but no difference in the $\mathrm{Cq}$ values of $B$. animalis subsp. lactis or $S$. thermophilus. While the Cq values of B. longum subsp. infantis and B. animalis subsp. lactis were lowest during the supplementation period, the $\mathrm{Cq}$ values of $S$. thermophilus were lowest post-supplementation.

Using logistic regression, we examined the association between key variables (including gestational age, birth weight, antibiotic exposure, age started supplementation and duration of supplementation) and the detection of the probiotic species over the whole study period in only those infants who received the probiotic (Table S3). Commencing probiotic supplementation within 5 days after birth was 
Table 3. Effect of probiotic supplementation on detection of probiotic species before, during and after supplementation

\begin{tabular}{|c|c|c|c|c|c|c|}
\hline & \multicolumn{2}{|c|}{ Probiotic } & \multicolumn{2}{|c|}{ Placebo } & \multirow{2}{*}{ OR $(95 \% \mathrm{CI})^{\star}$} & \multirow{2}{*}{$P$ value } \\
\hline Over whole study period & $\mathrm{n}=\mathbf{2 2 9}$ & $n=1178$ & $\mathrm{n}=\mathbf{2 3 0}$ & $n=1153$ & & \\
\hline B. animalis subsp. lactis & $222(97)$ & $666(57)$ & $89(39)$ & $128(11)$ & $10.2(8.35-12.5)$ & $<0.001$ \\
\hline B. longum subsp. infantis & $225(98)$ & $729(62)$ & $112(49)$ & $214(19)$ & $7.16(5.89-8.69)$ & $<0.001$ \\
\hline Before supplementation & $n=225$ & $n=341$ & $n=222$ & $n=338$ & & \\
\hline B. animalis subsp. lactis & $8(4)$ & $8(2)$ & $3(1)$ & $3(1)$ & $2.67(0.72-9.99)$ & 0.144 \\
\hline B. longum subsp. infantis & $13(6)$ & $13(4)$ & $8(4)$ & $8(2)$ & $1.62(0.67-3.88)$ & 0.282 \\
\hline S. thermophilus & $15(7)$ & $15(4)$ & $11(5)$ & $13(4)$ & $1.17(0.52-2.62)$ & 0.700 \\
\hline B. longum subsp. infantis & $222(99)$ & $582(93)$ & $82(37)$ & $123(20)$ & $53.1(35.6-79.4)$ & $<0.001$ \\
\hline S. thermophilus & $205(92)$ & $418(66)$ & $114(51)$ & $161(26)$ & $5.66(4.35-7.37)$ & $<0.001$ \\
\hline After supplementation & $n=132$ & $n=207$ & $n=127$ & $n=194$ & & \\
\hline B. animalis subsp. lactis & $84(64)$ & $105(51)$ & $63(49)$ & $77(40)$ & $1.59(1.05-2.41)$ & 0.030 \\
\hline B. longum subsp. infantis & $103(78)$ & $134(65)$ & $66(52)$ & $83(43)$ & $2.53(1.64-3.90)$ & $<0.001$ \\
\hline S. thermophilus & $120(90)$ & $178(86)$ & $112(88)$ & $159(82)$ & $1.40(0.76-2.57)$ & 0.283 \\
\hline
\end{tabular}

*GEE logistic regression clustered for multiple specimens from each infant.

†Number and percentage of infants who provided at least one specimen in which the target was detected.

‡Number and percentage of specimens in which the target was detected.

associated with increased detection of $B$. animalis subsp. lactis (OR: 1.20; 95\% CI: 1.02-1.41; $P=0.033$ ), B. longum subsp. infantis (OR: 1.28 ; 95\% CI: $1.09-1.49 ; P=0.002$ ) and S. thermophilus (OR: 1.45; 95\% CI: 1.19-1.77; $P<0.001$ ). Receiving 65 days or more of probiotic was associated with increased detection of $B$. animalis subsp. lactis (OR: 1.25; 95\% CI: 1.06-1.47; $P=0.008$ ), but not the other two species. Gestational age $\leq 28$ weeks was associated with decreased detection of S. thermophilus (OR: 0.73; 95\% CI: 0.59-0.90; $P=0.003)$, and low birth weight $(<1000 \mathrm{~g})$ was associated with decreased detection of both $S$. thermophilus (OR: 0.79; 95\% CI: 0.64-0.96; $P=0.020)$ and B. longum subsp. infantis
(OR: 0.84; 95\% CI: 0.72-0.99; $P=0.033$ ). Infants exposed to antibiotics were less likely to have detection of the three probiotic species, but this was only statistically significant for S. thermophilus (OR: 0.76; 95\% CI: 0.62-0.93; $P=0.007$ ). Breastmilk feeding rates in this cohort were high (97\%) [10] so we could not investigate if breast feeding was associated with detection of probiotic species.

In total, 127 specimens had both $16 \mathrm{~S}$ rRNA gene sequencing data and qPCR data available. There was substantial concordance for the detection of Bifidobacterium (91\%, kappa coefficient $=0.79, P<0.001$, Table 4).

Table 4. Concordance of $\mathrm{PPCR}$ and $16 \mathrm{~S}$ rRNA gene sequencing for detection of Bifidobacterium

\begin{tabular}{|c|c|c|c|c|}
\hline & & & $\mathbf{P C R}^{*}$ & \\
\hline 16S rRNA gene sequencing $\dagger$ & Not detected & 30 & 4 & 34 \\
\hline & Detected & 7 & 86 & 93 \\
\hline & Column totals & 37 & 90 & 127 \\
\hline
\end{tabular}

Concordance $91 \%$, kappa coefficient $=0.79, P<0.001$.

${ }^{*}$ Genus-specific primers for Bifidobacterium targeting the 16S-23S rRNA intergenic spacer were used, and Bifidobacterium was considered detected by qPCR if Cq value <45. $\dagger$ †ifidobacterium was considered detected by $16 \mathrm{~S}$ rRNA gene sequencing if any sequence reads were assigned to the genus Bifidobacterium. 


\section{DISCUSSION}

In this study we investigated the impact of probiotic supplementation with $B$. longum subsp. infantis BB-02, S. thermophilus TH-4 and B. animalis subsp. lactis BB-12 on the gut microbiota of a subset of infants who participated in the ProPrems RCT $[9,10]$. Our results indicate that the probiotic combination increases the detection of B. longum subsp. infantis, B. animalis subsp. lactis and $S$. thermophilus in the gut microbiota of preterm infants during supplementation. While detection of B. longum subsp. infantis and B. animalis subsp. lactis was increased post-supplementation in infants who received the probiotic combination, there was no difference in the detection of $S$. thermophilus post-supplementation between the probiotic and placebo groups (Table 3). These data extend the existing 16S rRNA gene sequencing data from this cohort [12] by providing subspecies-level resolution from a large number of infants. Importantly, our results suggest that commencing probiotics closer to birth may be important for ensuring long-term colonization.

Our findings are consistent with previous studies that report increased detection of probiotic strains in probioticsupplemented infants compared to controls. A small longitudinal study investigating the gut microbiota of only seven infants supplemented with Infloran (a probiotic containing B. bifidum and Lactobacillus acidophilus) and three control infants found, using qPCR assays, that B. bifidium was increased in probiotic-supplemented infants during and after supplementation [19]. L. acidophilus was rarely detected in either probiotic or control infants [19]. The PiPs study, a large RCT ( $n=1310$ preterm infants) of the probiotic $B$. breve BBG-001, found that $84 \%$ of probiotic-supplemented infants were colonized with $B$. breve BBG-001 by strain-specific qPCR at 2 weeks of age compared to $35 \%$ of control infants [20]. Additionally, using culture methods, $B$. breve was detected in the stool of $84 \%$ of probiotic infants compared to $49 \%$ of placebo infants at 36 weeks of postmenstrual age [20]. Using a species-specific qPCR assay, Patole et al. [21] found that $B$. breve was detected in $91 \%(n=67 / 74)$ of infants supplemented with B. breve M-16V vs. $38 \%(n=25 / 66)$ of control infants 3 weeks after supplementation.

The presence of the probiotic species during supplementation is not necessarily confirmation that colonization of these species has occurred: rather it may simply represent detection of ingested probiotic that has passed through the gastrointestinal tract. Infants in our study provided specimens for up to 23 months post-supplementation, with the majority of specimens collected 6-12 months after ending supplementation (Table S1). Increased detection of probiotic species post-supplementation as demonstrated in our study (for B. longum subsp. infantis and B. animalis subsp. lactis) and previous studies [19-22] suggests long-term colonization. B. longum subsp. infantis had a relatively lower Cq post-supplementation compared to $B$. animalis subsp. lactis, suggesting that $B$. longum subsp. infantis may be more effective at colonizing the gut. This is supported by a two-part study of 17 infants that reported increased faecal
Bifidobacterium (by $16 \mathrm{~S}$ rRNA gene sequencing) in infants supplemented with $B$. longum subsp. infantis compared to infants supplemented with B. animalis subsp. lactis [23], but this was a small study with limited samples collected post-supplementation.

There is limited information regarding the optimal time to start probiotics; however, our results suggest that commencing shortly after birth may be important for colonization (Table S3). In addition, we found that early gestational age $(<28$ weeks), low birth weight $(<1000 \mathrm{~g})$ and antibiotic use were each associated with decreased detection of one or more of the probiotic species. Consistent with this, the PiPs study reported that in infants randomized to probiotic, increasing gestational age was associated with increased colonization and antibiotic use was associated with decreased colonization [20]. Early gestational age, low birth weight and antibiotic use are known to influence the gut microbiota composition [24], and are characteristics of a higher risk group of premature infants. It is possible that the negative association between these factors and probiotic colonization is a result of the immaturity of the gastrointestinal tract in these at-risk infants. Importantly, antibiotic use has been shown to reduce the abundance of beneficial bacteria including Bifidobacterium species and increase the abundance of potentially pathogenic bacteria in preterm infants [25]. Thus it is possible that these alterations to the gut microbiota impede colonization of the probiotic species.

S. thermophilus was the most prevalent species detected postsupplementation and no difference in detection was observed between probiotic and placebo-supplemented infants (Table 3). Most specimens collected post-supplementation were collected at 6 and 12 months corrected age, when infants had probably commenced a solid food diet. S. thermophilus is commonly used in the production of yoghurt and some cheeses [26], so the frequent detection of $S$. thermophilus post-supplementation in both allocation groups may suggest it was acquired through diet.

There are limitations to this study. First, the qPCR assays used were not strain-specific. As a result, we cannot be certain that the B. longum subsp. infantis, B. animalis subsp. lactis and $S$. thermophilus detected were the probiotic strains. However, the increased detection of probiotic species in probioticsupplemented infants compared to controls during the supplementation period suggests that the probiotic strains were present in these infants. Second, the PCR primers used for detection of S. thermophilus also detect S. salivarius [27]. A whole genome or metagenomic approach would provide higher specificity for detecting the probiotic strains and may reveal if genomic differences in B. longum subsp. infantis, $B$. lactis and S. thermophilus exist between probiotic and control infants. Finally, the detection of the probiotic species in samples collected prior to supplementation could indicate some cross-colonization took place while the infants were in the neonatal unit, which has previously been reported $[20,28-30]$. 


\section{CONCLUSION}

We report that probiotic supplementation with $B$. longum subsp. infantis $\mathrm{BB}-02, S$. thermophilus $\mathrm{TH}-4$ and $B$. animalis subsp. lactis BB-12 enhances the presence of B. longum subsp. infantis, B. animalis subsp. lactis and S. thermophilus in the gut microbiota of preterm infants during supplementation. We followed infants for up to 23 months post-supplementation and found increased detection of both Bifidobacterium probiotic species in infants randomized to probiotic compared to placebo infants, which suggests that probiotic use may result in long-term colonization. Importantly, we report that commencing probiotic supplementation shortly after birth may be important for improved long-term colonization of probiotic species.

\section{Funding information}

Financial support was provided by National Health and Medical Research Council of Australia (project grant 454629), The Royal Women's Hospital Foundation, Melbourne, Australia, and The Angior Family Foundation, Melbourne, Australia.

\section{Acknowledgements}

Members of The ProPrems Study Group are as follows: ProPrems Steering Group - S.M. Garland (principal investigator), S.E. Jacobs (chief investigator), J.M. Tobin (chief investigator), S.N. Tabrizi (chief investigator), M. Pirotta (chief investigator), S. Donath (associate investigator), G.F. Opie (associate investigator), M.L.K. Tang (associate investigator), C.J. Morley (associate investigator); Participating Hospitals and Investigators - S.M. Garland, S.E. Jacobs, C.J. Morley, S.N. Tabrizi, L. Hickey, The Royal Women's Hospital; G.F. Opie, Mercy Hospital for Women; K. Tan, A. Lewis, A. Veldman, Monash Medical Centre; M.L.K. Tang, Royal Children's Hospital, all in Melbourne, Australia; J. Travadi, I.M.R. Wright, John Hunter Children's Hospital; D.A. Osborn, Royal Prince Alfred Hospital; J. Sinn, Royal North Shore Hospital; J. Levison, J.A. Stack, Liverpool Hospital, all in New South Wales, Australia; A.G. DePaoli, Royal Hobart Hospital, Tasmania, Australia; N.C. Austin, B.A. Darlow, Christchurch Women's Hospital, University of Otago, Christchurch, New Zealand; J.M. Alsweiler, M.J Buksh, Auckland City Hospital and The Liggins Institute, University of Auckland, Auckland, New Zealand.

\section{Author contributions}

E. L. P., performed formal analysis, visualization, interpretation of data and writing - original draft preparation. J. A. D., performed investigation, interpretation of data and writing - original draft preparation. S. M. G. obtained funding, provided resources, and performed supervision, interpretation of data and writing - review and editing. S. E. J., performed interpretation of data and writing - review and editing. J. S., performed interpretation of data and writing - original draft preparation. G. L. M., performed interpretation of data, supervision and writing - original draft preparation. S. M. G. and S. E. J., contributed equally to the coordination of the ProPrems trial from which the samples used in this sub-study were collected.

\section{Conflicts of interest}

The authors declare that there are no conflicts of interest

\section{Ethical statement}

ProPrems was conducted in tertiary perinatal hospitals in Australia and New Zealand and approved by the Human Research Ethics Committees at each site. The initial approval was granted by The Royal Women's Hospital HREC. The RWH project approval number is 06/31. Written informed consent was obtained from parents or guardians prior to any study procedures.

\section{References}

1. Cilieborg MS, Boye M, Sangild PT. Bacterial colonization and gut development in preterm neonates. Early Hum Dev 2012;88:S41-9.
2. Matamoros S, Gras-Leguen C, LeVacon F, Potel G, de La Cochetiere MF. Development of intestinal microbiota in infants and its impact on health. Trends Microbiol 2013;21:167-173.

3. Di Gioia D, Aloisio I, Mazzola G, Biavati B. Bifidobacteria: their impact on gut microbiota composition and their applications as probiotics in infants. Appl Microbiol Biotechnol 2014;98:563-577.

4. Morelli L, Capurso L. FAO/WHO guidelines on probiotics: 10 years later. J Clin Gastroenterol 2012;46:S1-2.

5. Aceti A, Gori D, Barone G, Callegari ML, Di Mauro A, et al. Probiotics for prevention of necrotizing enterocolitis in preterm infants: systematic review and meta-analysis. Ital J Pediatr 2015:41:89.

6. AlFaleh K, Anabrees J. Probiotics for prevention of necrotizing enterocolitis in preterm infants. Cochrane Database Syst Rev 2014:4:CD005496.

7. Aceti A, Maggio L, Beghetti I, Gori D, Barone G, et al. Probiotics prevent late-onset sepsis in human milk-fed, very low birth weight preterm infants: Systematic review and meta-analysis. Nutrients 2017:9.

8. Deshpande G, Jape G, Rao S, Patole S. Benefits of probiotics in preterm neonates in low-income and medium-income countries: a systematic review of randomised controlled trials. BMJ Open 2017:7:e017638.

9. Garland SM, Tobin JM, Pirotta M, Tabrizi SN, Opie G, et al. The ProPrems trial: investigating the effects of probiotics on late onset sepsis in very preterm infants. BMC Infect Dis 2011;11:210.

10. Jacobs SE, Tobin JM, Opie GF, Donath S, Tabrizi SN, et al. Probiotic effects on late-onset sepsis in very preterm infants: a randomized controlled trial. Pediatrics 2013;132:1055-1062.

11. Plaza-Diaz J, Ruiz-Ojeda FJ, Gil-Campos M, Gil A. Mechanisms of action of probiotics. Adv Nutr 2019;10:S49-S66.

12. Plummer EL, Bulach DM, Murray GL, Jacobs SE, Tabrizi SN, et al. Gut microbiota of preterm infants supplemented with probiotics: sub-study of the ProPrems trial. BMC Microbiol 2018;18:184.

13. Tobin JM, Garland SM, Jacobs SE, Pirotta M, Tabrizi SN. Rapid assay to assess colonization patterns following in-vivo probiotic ingestion. BMC Res Notes 2013;6:252.

14. Haarman M, Knol J. Quantitative real-time PCR assays to identify and quantify fecal Bifidobacterium species in infants receiving a prebiotic infant formula. Appl Environ Microbiol 2005;71:2318-2324.

15. Sim K, Cox MJ, Wopereis H, Martin R, Knol J, et al. Improved detection of bifidobacteria with optimised 16S rRNA-gene based pyrosequencing. PLoS One 2012;7:e32543.

16. Caporaso JG, Kuczynski J, Stombaugh J, Bittinger K, Bushman FD, et al. QIIME allows analysis of high-throughput community sequencing data. Nat Methods 2010;7:335-336.

17. Edgar RC. Search and clustering orders of magnitude faster than BLAST. Bioinformatics 2010;26:2460-2461.

18. Quast C, Pruesse E, Yilmaz P, Gerken J, Schweer T, et al. The SILVA ribosomal RNA gene database project: improved data processing and web-based tools. Nucleic Acids Res 2013;41:D590-6.

19. Abdulkadir B, Nelson A, Skeath T, Marrs EC, Perry JD, et al. Routine use of probiotics in preterm infants: Longitudinal impact on the microbiome and metabolome. Neonatology 2016;109:239-247.

20. Costeloe K, Hardy P, Juszczak E, Wilks M, Millar MR. Probiotics in preterm infants study collaborative $G$. Bifidobacterium breve BBG-001 in very preterm infants: A randomised controlled phase 3 trial. Lancet 2016;387:649-660.

21. Patole S, Keil AD, Chang A, Nathan E, Doherty D, et al. Effect of Bifidobacterium breve M-16V supplementation on fecal bifidobacteria in preterm neonates--a randomised double blind placebo controlled trial. PLoS One 2014;9:e89511.

22. van Best N, Trepels-Kottek S, Savelkoul P, Orlikowsky T, Hornef MW, et al. Influence of probiotic supplementation on the developing microbiota in human preterm neonates. Gut Microbes 2020;12:1-16.

23. Underwood MA, Kalanetra KM, Bokulich NA, Lewis ZT, Mirmiran M, et al. A comparison of two probiotic strains of bifidobacteria in premature infants. J Pediatr 2013;163:1585-1591. 
24. Henderickx JGE, Zwittink RD, van Lingen RA, Knol J, Belzer C. The preterm gut microbiota: An inconspicuous challenge in nutritional neonatal care. Front Cell Infect Microbiol 2019;9:85.

25. Gibson MK, Crofts TS, Dantas G. Antibiotics and the developing infant gut microbiota and resistome. Curr Opin Microbiol 2015;27:51-56.

26. Delorme C. Safety assessment of dairy microorganisms: Streptococcus thermophilus. Int J Food Microbiol 2008;126:274-277.

27. Furet JP, Quenee P, Tailliez P. Molecular quantification of lactic acid bacteria in fermented milk products using real-time quantitative PCR. Int J Food Microbiol 2004;97:197

28. Hickey L, Garland SM, Jacobs SE, O'Donnell CP, Tabrizi SN, et al. Cross-colonization of infants with probiotic organisms in a neonatal unit. J Hosp Infect 2014;88:226-229.
29. Kitajima H, Sumida Y, Tanaka R, Yuki N, Takayama H, et al. Early administration of Bifidobacterium breve to preterm infants: randomised controlled trial. Arch Dis Child Fetal Neonatal Ed 1997;76:F101-7.

30. Yousuf El, Carvalho M, Dizzell SE, Kim S, Gunn E, et al. Persistence of suspected probiotic organisms in preterm infant gut microbiota weeks after probiotic supplementation in the NICU. Front Microbiol 2020;11:574137.

31. Matsuki T, Watanabe K, Tanaka R, Fukuda M, Oyaizu H. Distribution of bifidobacterial species in human intestinal microflora examined with $16 \mathrm{~S}$ rRNA-gene-targeted species-specific primers. Appl Environ Microbiol 1999;65:4506-4512.

32. Ventura M, Reniero R, Zink R. Specific identification and targeted characterization of Bifidobacterium lactis from different environmental isolates by a combined multiplex-PCR approach. Appl Environ Microbiol 2001;67:2760-2765.

\section{Five reasons to publish your next article with a Microbiology Society journal}

1. The Microbiology Society is a not-for-profit organization.

2. We offer fast and rigorous peer review - average time to first decision is 4-6 weeks.

3. Our journals have a global readership with subscriptions held in research institutions around the world.

4. $80 \%$ of our authors rate our submission process as 'excellent' or 'very good'.

5. Your article will be published on an interactive journal platform with advanced metrics.

Find out more and submit your article at microbiologyresearch.org. 


\section{University Library}

\section{- M M I N E R VA A gateway to Melbourne's research publications}

Minerva Access is the Institutional Repository of The University of Melbourne

Author/s:

Plummer, EL;Danielewski, JA;Garland, SM;Su, J;Jacobs, SE;Murray, GL

Title:

The effect of probiotic supplementation on the gut microbiota of preterm infants.

Date:

2021-08

Citation:

Plummer, E. L., Danielewski, J. A., Garland, S. M., Su, J., Jacobs, S. E. \& Murray, G. L. (2021). The effect of probiotic supplementation on the gut microbiota of preterm infants.. J Med Microbiol, 70 (8), pp.001403-. https://doi.org/10.1099/jmm.0.001403.

Persistent Link:

http://hdl.handle.net/11343/294186

License:

CC BY 